\title{
Induced loudness reduction as a function of frequency difference between test tone and inducer
}

\author{
Jeremy Marozeau and Michael Epstein \\ Northeastern University, Boston, Massachusetts
}

\begin{abstract}
When a high-intensity tone (inducer) is followed by a moderate-intensity tone (test tone), the loudness of the latter is reduced. This phenomenon, called induced loudness reduction (ILR), depends on the frequency separation of the two tones; as the difference in frequency increases, the amount of ILR decreases. However, the precise course of this decrease is not well known. This article presents two experiments that address this question. In the first experiment, the amount of loudness reduction produced by a $2.5-\mathrm{kHz} 80-\mathrm{dB}-\mathrm{SPL}$ inducer was measured with the frequency of the test tone swept from $800 \mathrm{~Hz}$ to $6 \mathrm{kHz}$. In the second experiment, the amount of ILR was measured with the same inducer and with test tones set at 2, 2.5, 3, and $4 \mathrm{kHz}$. Both experiments show that some ILR occurs at frequency separations as wide as four critical bands.
\end{abstract}

Psychoacoustical experiments examining loudness have always been prone to unexplained variability, typically attributed to biases resulting from subtle procedural differences between experiments (see Poulton, 1989, for a review). Very often, these biases are explained using high-level perceptual concepts such as learning effects, range effects, or subject preferences. More recently, a number of studies have examined the precise contexts in which some of these types of discrepancies appear and have yielded specific patterns. In many loudness experiments, the loudness of a sound may be reduced when it is preceded by a higher intensity sound (inducer) at a close frequency, with an equal or longer duration. This phenomenon, initially noted as a slippery-context effect (Marks, 1992), has more recently been termed induced loudness reduction (ILR) or loudness recalibration (Arieh \& Marks, 2001; Mapes-Riordan \& Yost, 1997, 1999; Marks, 1994; Scharf, Buus, \& Nieder, 2002).

ILR is a pervasive effect whose origin has been debated, and it has the potential to result in unexpected effects on data collected during loudness experiments involving the presentation of a range of levels, particularly any experiment that groups moderate- and high-level presentations. Notably, it has been shown that the amount of ILR is greater at moderate levels (Mapes-Riordan \& Yost, 1999). Because the effects of ILR accumulate over time, results obtained in the early portions of experiments, prior to significant sound exposure, may differ markedly from results obtained in later portions of experiments (Arieh, Kelly, \& Marks, 2005). Epstein (2007) examined the results of a magnitude estimation study by Hellman and Zwislocki (1963). The magnitude estimation data indicated that over the course of the experiment, loudness changed more for moderate-level sounds than it did for low- and high-level sounds. The elucidation of this effect raises important questions regarding the "true" nature of the loudness function, particularly the shallow, compressive region seen at moderate levels, the origin of which is still in debate (Braida \& Durlach, 1972; Buus, Florentine, Poulsen, \& Müsch, 1999; Epstein \& Florentine, 2005; Florentine \& Epstein, 2006; Parker \& Schneider, 1994; Schneider \& Parker, 1990; Ward, Armstrong, $\&$ Golestani, 1996). In addition, loudness calculation and modeling for sound has typically been done a priori, using only the present physical stimulus parameters. In fact, the effects of ILR provide significant motivation to generate loudness models that include not only immediate physical parameters, but also long-term context parameters.

It is, however, difficult to model the effects of ILR on a particular experiment, because of the dependence on several factors (see Epstein, 2007, for a review), including (1) tone levels (Mapes-Riordan \& Yost, 1999), (2) frequency separation between inducer and test tones (Marks, 1994), (3) durations of inducer and test tones (Nieder, Buus, Florentine, \& Scharf, 2003), (4) time separation between inducer and test tones (Arieh \& Marks, 2003), (5) number of exposures to inducers (Arieh et al., 2005), and (6) individual differences (Epstein \& Gifford, 2006; Wagner \& Scharf, 2006).

Previously, the effects of frequency on ILR have not been thoroughly examined. Marks and Warner (1991) showed that loudness functions are affected by the frequency-specific context of measurements, and Marks (1994) showed that loudness matches between tones that differ in frequency are affected by the frequency separation. These studies imply that an inducer with a frequency that is nearly the same as that of the test tone causes maximal ILR and an inducer that is at a frequency that is very different from that of the test tone does not cause ILR. However, the relationship between the frequencies of the

J. Marozeau, j.marozeau@neu.edu 
inducer and the test tone and the amount of ILR has not been directly tested and is not well understood for smallto-moderate frequency spacings. Many previous loudness experiments have been based on loudness matches between sounds at nearby frequencies. In order to determine what possible influence ILR may have had on those data, it is necessary to gain a further understanding of how ILR changes as a function of frequency spacing. Therefore, in the present study, the amount of ILR was examined as a function of the frequency separation between the inducer and the test tones. The first experiment, a modified equalloudness-tracking Békésy procedure, examined this relationship by sweeping in small frequency increments. Then a follow-up experiment examined the amount of ILR at specific frequency spacings.

The frequency spacings are defined in this article in terms of equivalent rectangular bandwidth number (ERBn; ANSI, 2007). The frequency is related to the ERBn by the following formula:

$$
\mathrm{ERBn}=21.366 \log _{10}(0.004368 f+1) .
$$

When two tones are presented simultaneously, different perceptual phenomena will occur, depending on whether the frequency separation of the tones is smaller or greater than a certain value. This value is known as a critical band. The ERBn can be used to calculate whether the two tones are within the same critical band. If the frequency difference in ERBn is smaller than one, the tones are in the same critical band. If the difference is greater than one, the tones are in different critical bands.

\section{EXPERIMENT 1}

\section{Method}

Procedure. A modified Békésy tracking procedure was used to evaluate the amount of ILR as a function of the frequency separation. The experiment was divided into two conditions: the baseline and the inducer conditions. In the baseline condition, a test tone and a comparison tone were presented during each trial. In the inducer condition, an inducer was presented prior to the test tone and the comparison tone. The stimuli were all pure tones with equivalent rectangular durations of $200 \mathrm{msec}$ (i.e., they had the same energy as a rectangular temporal envelope tone). The tones had 6.67-msec Hanning-window rises and falls and $195-\mathrm{msec}$ steady-state durations. The tones were presented monaurally to the right ear for all the listeners. The inducer was a $2500-\mathrm{Hz}$ tone presented at $80 \mathrm{~dB}$ SPL. The test tone was presented at a fixed level of $70 \mathrm{~dB}$ SPL. It started $1,250 \mathrm{msec}$ after the inducer in the inducer condition and $1,450 \mathrm{msec}$ after the previous trial in the baseline condition. The frequency of the test tone varied from $800 \mathrm{~Hz}$ to $6.2 \mathrm{kHz}$, with a 0.1 ERBn increase in frequency at each trial. The comparison tone, which started $550 \mathrm{msec}$ after the test tone, was presented at a fixed frequency of $500 \mathrm{~Hz}$. The level of the comparison tone started at $72 \mathrm{~dB}$ SPL and varied according to the judgment made in the previous trial. The starting level was selected in order to approximately set the comparison tone to a loudness that was the same as that of the test tone at the beginning of the session (ANSI, 2004). A 1,600-msec silence was left between the end of the comparison tone and the beginning of the next trial, resulting in trials totaling $4 \mathrm{sec}$ in length.

The task of the listener was to judge whether the test or the comparison tone was louder, while ignoring the inducer (if presented). If the listener judged that the test tone was louder than the comparison tone, the level of the comparison was increased by $1.5 \mathrm{~dB}$ in the following trial. Otherwise, the comparison tone level was decreased by
$1.5 \mathrm{~dB}$. In order to keep the presentation timing carefully controlled, the next trial started automatically $1,600 \mathrm{msec}$ after the comparison tone. If the listener did not give any answer during this time, the judgment made on the previous trial was used to set the new level of the comparison tone. The session stopped when the frequency of the test tone reached $6.2 \mathrm{kHz}$. The total number of trials was 171, which lasted $11 \mathrm{~min} 24 \mathrm{sec}$.

The baseline condition was identical to the inducer condition, except that the inducer was attenuated so as to be inaudible. Each condition was repeated three times, each time on a different day. Each day, one baseline and one inducer condition were run. The baseline condition was always first, in order to reduce the ILR effect on this condition. The listeners took a 15-min break between the sessions to prevent undesired ILR carryover effects (Epstein \& Gifford, 2006).

Listeners. Ten listeners ( 6 of them female and 4 male) participated in this experiment. They ranged in age from 20 to 45 years old, with a mean age of 27 years. All the listeners had audiometric thresholds of less than $20 \mathrm{~dB}$ HL (ANSI, 2004) at octave frequencies from 250 to $8000 \mathrm{~Hz}$ in both ears, when measured clinically, and had medical histories consistent with normal hearing. The listeners were members of the laboratory or volunteers, and all of them had previous experience making equal-loudness judgments. Volunteers from outside the laboratory were paid $\$ 10 / \mathrm{h}$ for their participation.

Apparatus. The listeners were seated in a sound-attenuating booth. A PC-compatible computer with a signal processor (TDT AP2) generated the stimuli, recorded the listener's responses, and

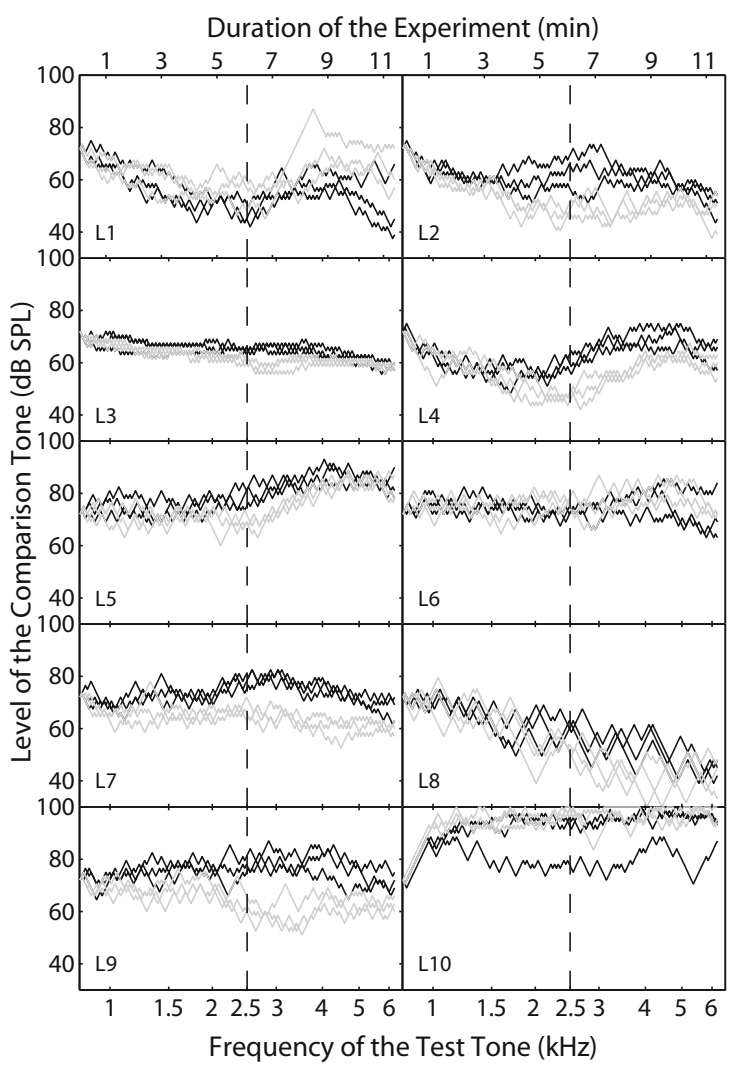

Figure 1. The level of the comparison tone as a function of the frequency/time matched to the loudness of a $2500-\mathrm{Hz}$ test tone at $60 \mathrm{~dB}$ SPL in each of the three repetitions of the baseline (dark lines) and the inducer (light lines) conditions for 10 listeners in Experiment 1. 
executed the adaptive procedure. The sample rate was $48 \mathrm{kHz}$. The output of the 16-bit D/A converter was attenuated, low-pass filtered, and attenuated again and led to a headphone amplifier, which fed one earphone of the Sony MDR-V6 headset.

\section{Results}

Individual data. Figure 1 shows the individual results for the six sessions. The three thick black lines represent the level of the comparison as a function of time (or frequency of the test tone) in the baseline condition. For clarity's sake, it is worth noting that these lines indicate how the loudness of a tone with a fixed level varies with frequency. These lines are different from equal-loudness contours, which represent the level of tones with fixed loudnesses as a function of frequency. The small difference between these lines indicates that the amount of intrasubject variation is quite small (i.e., the listeners are generally consistent). The average standard deviation between these lines ranges from 2 for Listener L3 to 7.9 for Listener L10.

The three light lines represent the level of the comparison tone for the inducer condition. The listeners are also generally consistent in the inducer condition, with standard deviations ranging from 1.4 for Listener L3 to 5.5 for Listener L1. However, two main inconsistencies are apparent. Listener L1 shows an abnormal increase after $3000 \mathrm{~Hz}$. In a postexperiment interview, she remembered falling asleep in the middle of this session. During this period, the lack of response was interpreted by the procedure as a continuous repetition of the last response, which led to the constant increase in level. One of L10's sessions was highly inconsistent with his other sessions. No clear explanation was found for this behavior.

The amount of ILR can be measured by comparing the baseline and the inducer conditions. Figure 1 shows that the inducer condition has the general tendency to be lower than the baseline. The average level in the inducer condition is $3.4 \mathrm{~dB}$ below the average level in the baseline condition. In order to estimate whether this difference was significant, a two-way repeated measures ANOVA was run for each listener, with frequency $(d f=170)$ and condition $(d f=1)$ as the main factors. For every subject, the effect of frequencies was significant $(p<.01)$. For 7 subjects out of 10 (L2, L3, L4, L5, L7, L8, and L9), the interaction between frequency and condition was significant $(p<$ $.01)$. These results show that (1) the amount of ILR was significant and (2) it was dependent on the frequency of the test tone. For the 3 other subjects (L1, L6, and L10), no significant effect was found for the condition or its interactions. Therefore, they showed no significant ILR, and they were excluded from the rest of the analysis. In previous ILR experiments, it has not been uncommon to find listeners that did not show any ILR (Epstein, 2007).

Figure 2 shows the amount of ILR for each listener, estimated as the average of the three inducer conditions subtracted from the average of the three baseline conditions. The results were fit using an eighth-order polynomial. The shaded area represents the average plus and minus the standard deviation at each frequency. All the listeners showed a similar pattern of results. The amount

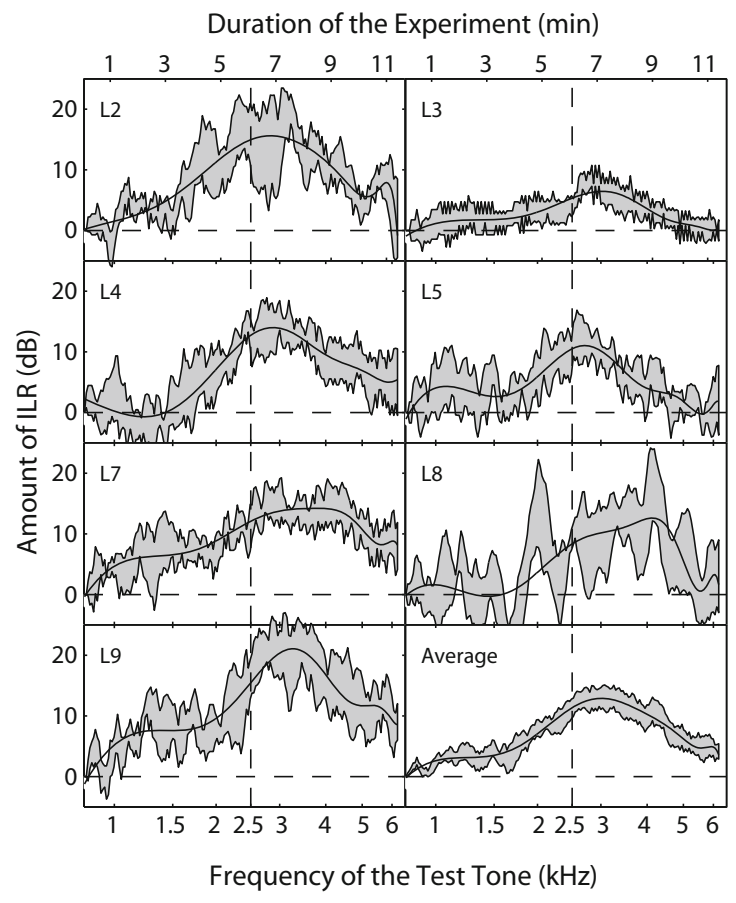

Figure 2. The amount of induced loudness reduction (ILR) caused by a $2500-\mathrm{Hz}$ inducer as a function of frequency for each of the 7 listeners who exhibited some amount of ILR in Experiment 1 and the average for those listeners. The shaded area represents the average plus and minus one standard deviation (individual listeners) or plus and minus one standard error of the mean (average) at each frequency.

of ILR started at zero, because every session started at the same level, increased slowly to reach a maximum between 2.5 and $3 \mathrm{kHz}$, and then decreased to a level close to zero at the highest frequencies tested. The maximum amount of ILR for each listener ranged from 6.5 for L3 to 21 for L9. Surprisingly, the maximum ILR occurred for most of the listeners between 2.7 and $3.3 \mathrm{kHz}$, and not at the frequency of the inducer $(2.5 \mathrm{kHz})$.

Average data. The last panel in Figure 2 shows the average amount of ILR as function of frequency for the 7 listeners who showed a significant amount of ILR. The average shows three striking results. First, the frequency range of the effect for a $2.5-\mathrm{kHz}$ inducer is much wider than 1 critical band. The amount of ILR is greater than $5 \mathrm{~dB}$ between 1782 and $5300 \mathrm{~Hz}$. This bandwidth corresponds to 9.4 critical bands (ERBn). Second, the effect is slightly asymmetrical. The amount of ILR rises from $5 \mathrm{~dB}$ to the maximum in 4.4 ERBn, then decreases from the maximum to $5 \mathrm{~dB}$ in $5 \mathrm{ERBn}$. Therefore, a high-frequency tone is much more likely to be affected by a lower frequency inducer than the opposite. Third, the maximum amount of ILR is $12.9 \mathrm{~dB}$ and occurs when the frequency of the test tone is $3 \mathrm{kHz}$. This is surprising because this frequency is higher than that of the inducer. Several possible explanations could account for this last striking result, but first, it was necessary to determine whether this effect resulted 
from a bias introduced by the methodology, because the frequency of the test tone was always increasing with each presentation. In order to test this possibility, a second experiment was performed.

\section{EXPERIMENT 2}

\section{Method}

Procedure. This experiment was designed to reproduce previous ILR experiments (Arieh \& Marks, 2003; Mapes-Riordan \& Yost, 1999), while keeping the same parameters for the inducer and the comparison tones as in the present Experiment 1. The listeners were asked to adjust the level of a comparison tone to match the loudness of a fixed frequency test tone in a two-interval, two-alternative forced choice paradigm with four interleaved tracks. In the baseline condition, only the test and the comparison tones were presented. In the inducer condition, a series of 12 inducers were presented prior to the first trial. Then, before each test tone, 1 inducer was presented. The time separations between the tones were the same as those in Experiment 1, except that subsequent trials did not automatically begin. Each new trial began $110 \mathrm{msec}$ after the listener's response. Each condition was divided into four blocks that differed only by the frequency of the test tone. The same types of stimuli as those used in Experiment 1 were used in Experiment 2. The inducer and the comparison tones were exactly the same; only the test tone differed. Instead of having the frequency of the test tone increase at every trial, the frequency was fixed inside a block. Four frequencies were used: $2000,2500,3000$, and $4000 \mathrm{~Hz}$, corresponding to separations of $-1.9,0,1.6$, and 4 ERBn from the $2500-\mathrm{Hz}$ inducer. The level of the comparison tone was adjusted according to a simple up-down method, with a step size of $5 \mathrm{~dB}$ until the second reversal and then $2 \mathrm{~dB}$ for seven reversals until the track ended. For each trial, the track was selected randomly from among those that had not yet ended. Equal loudness for each track was calculated as the average of the last four reversals. Each condition was repeated three times. The average of the 12 estimations per block was calculated as the final adjusted level. The amount of ILR was calculated as the difference between the baseline and the inducer conditions for the block frequency. Each condition was separated by at least a 15 -min break to prevent undesired ILR carryover effects (Epstein \& Gifford, 2006). The apparatus was the same as that used in Experiment 1.

Listeners. Nine listeners ( 5 of them female, 4 male) participated in the second experiment. They ranged in age from 20 to 30 years old, with a mean age of 25 years. All the listeners had audiometric thresholds less than $20 \mathrm{~dB}$ HL (ANSI, 2004) at octave frequencies from 250 to $8000 \mathrm{~Hz}$ in both ears, when measured clinically, and had medical histories that were consistent with normal hearing. The listeners were members of the laboratory or volunteers, and all of them had previous experience making equal-loudness judgments. Volunteers from outside the laboratory were paid $\$ 10 / \mathrm{h}$ for their participation. Among the 9 listeners, 6 also had participated in Experiment 1 . These 6 listeners included 1 of the 3 who had failed to show significant ILR in Experiment 1.

\section{Results}

Individual data. Figure 3 shows the individual responses for each listener. The listeners who participated in Experiment 1 are designated by the same number in Experiment 2. The listeners who did not participate are designated by a number greater than 10 . The lines represent the average adjusted level of the comparison tone as a function of the frequency of the test tone. The bars represent the standard deviations of the 12 tracks. The level for the inducer condition is always lower or the same (except for $\mathrm{L} 2$ at $4 \mathrm{kHz}, \mathrm{L} 3$ at $2 \mathrm{kHz}$, and $\mathrm{L} 11$ at $2 \mathrm{kHz}$ ), indicating a possible presence of ILR. The standard deviation

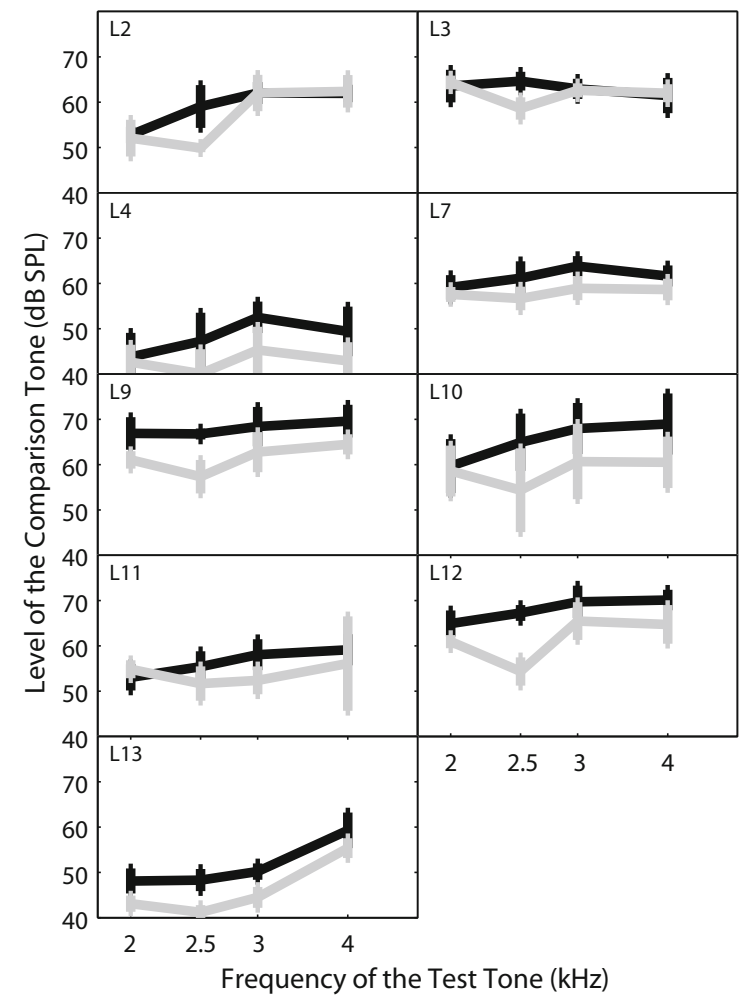

Figure 3. The level of the comparison tone as a function of the frequency matched to the loudness of a $2500-\mathrm{Hz}$ test tone at $60 \mathrm{~dB}$ SPL in the baseline (dark lines) and the inducer (light lines) conditions for 9 listeners in Experiment 2. The error bars show the standard deviations.

ranges from 1.2 for $\mathrm{L} 9$ at $2.5 \mathrm{kHz}$ to 6.7 for $\mathrm{L} 10$ at $4 \mathrm{kHz}$ in the baseline condition and from 0.9 for $\mathrm{L} 2$ at $2.5 \mathrm{kHz}$ to 10.41 for L11 at $4 \mathrm{kHz}$ in the inducer condition. The standard deviation is, on average, smaller than that for Experiment 1 , which should be expected because each condition was repeated 12 times, in contrast to the three repetitions in Experiment 1.

A set of ANOVAs, similar to those run in Experiment 1, was conducted. In this experiment, frequency was significant for all the listeners $(d f=3, p<.01$; except for L11, $p<.05$ ). In addition, all the listeners showed a significant effect of condition and/or an interaction between condition and frequency. Three listeners (L4, L7, and L13) showed only a significant effect of condition $(d f=1, p<.01)$, and 1 listener (L3) showed only a significant effect of the interaction $(d f=3, p<.01)$. All the other listeners showed a significant effect for both $(p<.01$; except for L9 and $\mathrm{L} 11$, interaction, $p<.05$ ). It is worth noting that $\mathrm{L} 10$, who did not show any significant ILR in Experiment 1, showed significant ILR in Experiment 2. The consistency of L10's data with those for the other listeners in Experiment 2 and the lack of consistency of L10's data with those for the other listeners in Experiment 1 suggest that L10 may have had some difficulty properly performing the task used in Experiment 1. 
The amount of ILR, shown in Figure 4, was calculated by subtracting the average level of the comparison tone in the baseline condition from the average level of the comparison tone in the inducer condition. The standard deviation of the amount of ILR was calculated by taking the square root of the variance average in the two conditions.

The maximum amount of ILR ranges from 4.9 for L7 to 13 for L12, consistent with Experiment 1. However, this time, for 6 out of 9 listeners (L2, L3, L9, L10, L12, and L13), the maximum occurs when the frequency of the test tone is the same as the frequency of the inducer. For 2 listeners (L4 and L7), the amount of ILR is about the same for 2.5- and 3-kHz test tones. For 1 listener (L11), the maximum amount of ILR occurs at $3 \mathrm{kHz}$.

A $t$ test was performed on each condition and each listener to evaluate whether the effect of ILR was significant. The results are represented in Figure 4. When the test showed no significant effect $(p>.01)$, the amount of ILR is represented by an open symbol, and when the effect is significant, ILR is represented by a filled symbol. ILR is significant for every listener at $2.5 \mathrm{kHz}$, for 7 listeners at $3 \mathrm{kHz}$, for 6 at $4 \mathrm{kHz}$, and only for 3 at $2 \mathrm{kHz}$.

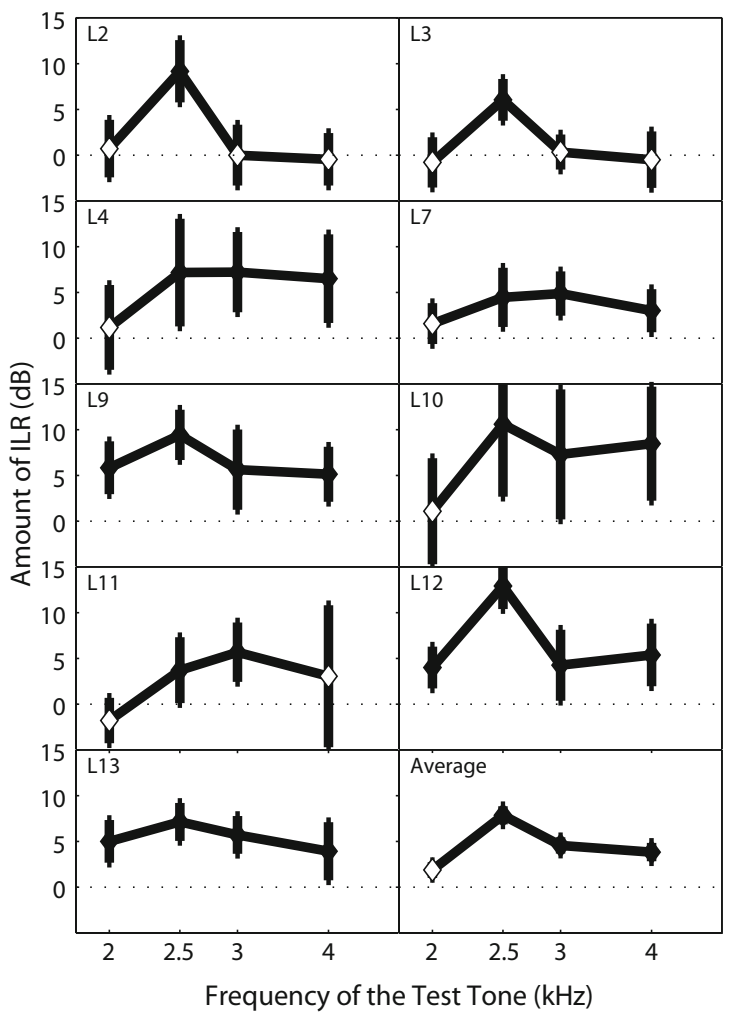

Figure 4. The amount of induced loudness reduction (ILR) caused by a $2500-\mathrm{Hz}$ inducer as a function of frequency for each of the 9 listeners in Experiment 2 and the average for those listeners. The error bars show the standard deviations (individual listeners) or the standard errors (average). The filled symbols represent a significant amount of ILR according to a $t$ test (individual listeners; $p<.01$ ) or an ANOVA (average; $p<.01$ ).
Average data. The last panel in Figure 4 shows the average amount of ILR as a function of frequency. Average results show maximum ILR at $2.5 \mathrm{kHz}$, with an amount of reduction of $7.8 \mathrm{~dB}$. The amount is within the range found in the literature. A two-way ANOVA was run for each frequency separately, with condition $(d f=1)$ and number of the repetition $(d f=11)$ as main factors. As is shown in the figure by an open symbol, the effect of ILR was not significant at $2 \mathrm{kHz}(p>.01)$ but was significant at $2.5 \mathrm{kHz}(p<.01), 3 \mathrm{kHz}(p<.01)$, and $4 \mathrm{kHz}(p<$ $.01)$. These results show that, on average, an inducer at $2.5 \mathrm{kHz}$ has no significant effect on tones at frequencies below $2 \mathrm{kHz}$ (1.9 ERBn) but can have an effect on tones at frequencies up to at least $4 \mathrm{kHz}(4 \mathrm{ERBn})$. This finding supports two of the results of Experiment 1: The effect of ILR is asymmetrical, and it is much wider than a critical band. However, it does not support the third finding in Experiment 1: The maximum amount of ILR does not occur at a frequency higher than the frequency of the inducer.

\section{DISCUSSION}

The results of both experiments demonstrate that ILR is a more pervasive effect, relative to frequency, than was previously believed. Specifically, an inducer may cause ILR on another tone that is at least 4 ERBn higher in frequency. The pattern also shows a wider spread of ILR toward higher frequencies than toward lower frequencies, similar to the spread of excitation on the basilar membrane (Moore \& Glasberg, 1987; Zwicker \& Fastl, 1990). Figure 5 shows an excitation pattern according to the ANSI standard loudness model (ANSI, 2007) for a $2.5-\mathrm{kHz}$ tone at $80 \mathrm{~dB}$ SPL. This similarity suggests that the amount of ILR is dependent on the amount of excitation on the basilar membrane. However, because of the contralateral effects of ILR, it has been argued that at least some of the effects of ILR are attributable to a central mechanism (Nieder, Buus, Cazals, \& Scharf, 2007). Therefore, more data are needed to determine whether this correlation is coincidental or the result of the same physiological mechanism.

The two experiments show some dissimilarity. In Experiment 1 , when the tracking procedure was used, the maximum amount of ILR occurred when the test tone frequency was higher than that of the inducer. Since this finding was not replicated in Experiment 2, it is likely that this finding was due to some shift in the ILR pattern that occurred as a result of the sweep rate of the test tone. It is clear that some delay between the subject's response and the compensatory level adjustment should be seen on the frequency scale; however, it is not clear to what degree the present results reflect only this factor. It would be necessary to study the effects of sweep rate on the ILR pattern in order to better understand this relationship. In addition, the frequency width of the effect of ILR was greater in Experiment 1 than in Experiment 2. Again, this may simply result from the data delay caused by the sweep or some other inherent difference between the procedures, similar to results seen in sweeping auditory threshold measurements (Miskiewicz, Buus, \& Florentine, 1994). 


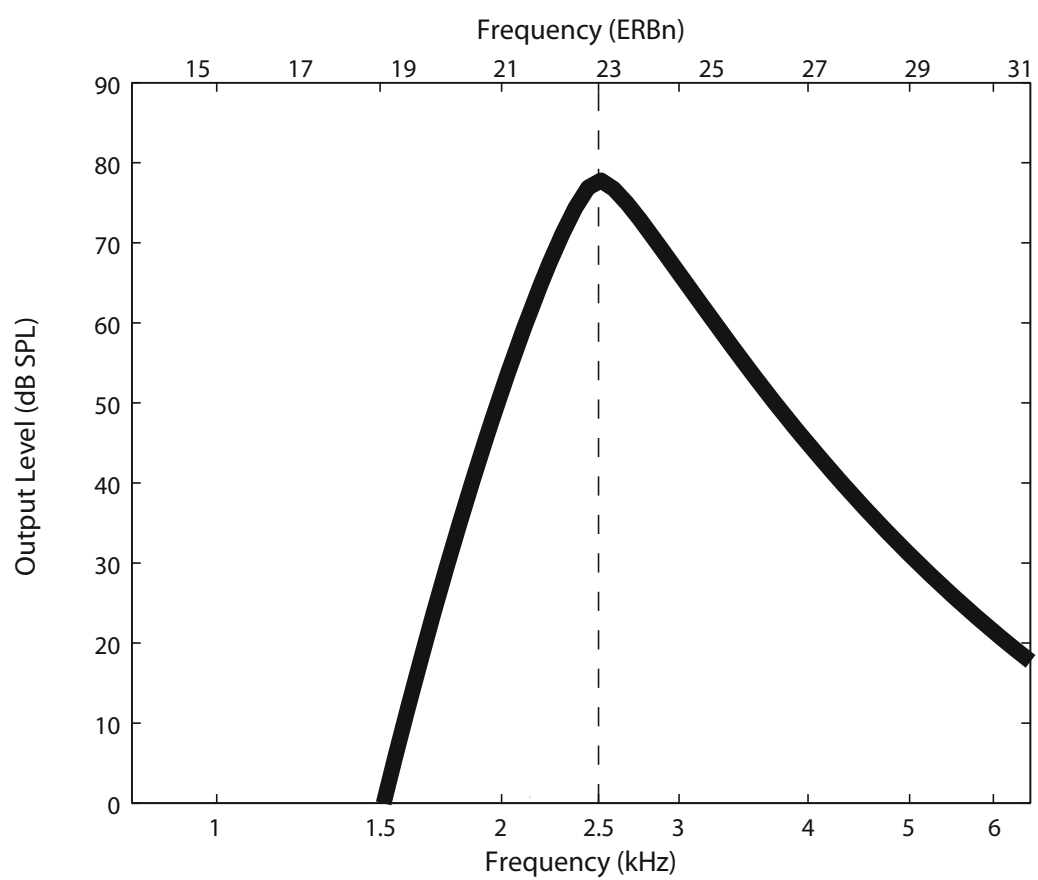

Figure 5. Excitation pattern of a $2.5-\mathrm{kHz}$ tone presented through headphones at $80 \mathrm{~dB}$ SPL according to the ANSI standard loudness model (ANSI, 2007).

The finding that ILR is more pervasive across frequency than was previously believed provides another guideline for the careful design of experiments in order to prevent undesired ILR effects. In addition, it may help explain some of the variability seen in loudness-matching and masking experiments that include tones that are relatively close in frequency.

These results suggest that a number of experimentsincluding, for example, those commonly used to measure equal-loudness contours - may have been affected by ILR. Most of the experiments deriving contours have used loudness-matching paradigms that required listeners to match the loudnesses of tones at different frequencies to a reference tone at $1 \mathrm{kHz}$ (see Suzuki \& Takeshima, 2004, for a review). The results from the present experiment suggest that the reference tone may have reduced the loudness of matched tones that were nearby in frequency. Therefore, the shape of the equalloudness contours may depend on the frequency of the reference tone.

\section{AUTHOR NOTE}

A portion of this work was presented at the 151 st meeting of the Acoustical Society of America. This research was supported by NIH/ NIDCD Grant R01DC02241 and the Swiss National Science Foundation. The authors thank Mary Florentine and Bertram Scharf for their support and helpful comments and Simona Ioffe for collecting the data. Correspondence concerning this article should be addressed to J. Marozeau, Department of Speech and Language Pathology and Audiology, Northeastern University, 360 Huntington Avenue, Boston, MA 02115 (e-mail: j.marozeau@neu.edu).

\section{REFERENCES}

ANSI (2004). American national standard specification for audiometers. ANSI S3.6-2004.

ANSI (2007). American national standard procedure for the computation of loudness of steady sounds. ANSI S3.4-2007.

Arieh, Y., Kelly, K., \& Marks, L. E. (2005). Tracking the time to recovery after induced loudness reduction [Letter]. Journal of the Acoustical Society of America, 117, 3381-3384.

ArIEH, Y., \& Marks, L. E. (2001). Recalibration of loudness: Sensory vs. decisional processes. In E. Sommerfeld, R. Kompass, \& T. Lachman (Eds.), Fechner Day 2001 (pp. 242-247). Berlin: Pabst.

Arieh, Y., \& MARKs, L. E. (2003). Time course of loudness recalibration: Implications for loudness enhancement. Journal of the Acoustical Society of America, 114, 1550-1556.

BraidA, L. D., \& DURLACH, N. I. (1972). Intensity perception: II. Resolution in one-interval paradigms. Journal of the Acoustical Society of America, 51, 483-502.

Buus, S., Florentine, M., Poulsen, T., \& Müsch, H. (1999). On the relation between peripheral compression and the growth of loudness. Journal of the Acoustical Society of America, 105, 1022.

EPSTEIN, M. (2007). An introduction to induced loudness reduction [Letter]. Journal of the Acoustical Society of America, 122, EL74-EL80.

Epstein, M., \& Florentine, M. (2005). A test of the equal-loudnessratio hypothesis using cross-modality matching functions. Journal of the Acoustical Society of America, 118, 907-913.

EPSTEIN, M., \& GiFFord, E. (2006). A potential carry-over effect in the measurement of induced loudness reduction. Journal of the Acoustical Society of America, 120, 305-309.

Florentine, M., \& Epstein, M. (2006). To honor Stevens and repeal his law (for the auditory system). In D. E. Kornbrot, R. M. Msetfi, \& A. W. MacRaein (Eds.), Fechner Day 2006 (pp. 37-42). International Society for Psychophysics, St. Albans, U.K.

Hellman, R. P., \& ZWISLOCKI, J. J. (1963). Monaural loudness function at $1000 \mathrm{cps}$ and interaural summation. Journal of the Acoustical Society of America, 35, 856-865. 
MAPES-RIORDAN, D., \& Yost, W. A. (1997). Loudness recalibration as a function of recalibration and comparison tone level. Journal of the Acoustical Society of America, 101, 3170.

Mapes-Riordan, D., \& Yost, W. A. (1999). Loudness recalibration as a function of level. Journal of the Acoustical Society of America, 106, 3506-3511.

MARKs, L. E. (1992). The slippery context effect in psychophysics: Intensive, extensive, and qualitative continua. Perception \& Psychophysics, 51, 187-198.

MARKS, L. E. (1994). "Recalibrating" the auditory system: The perception of loudness. Journal of Experimental Psychology: Human Perception \& Performance, 20, 382-396.

MARKS, L. E., \& WARNER, E. (1991). Slippery context effect and critical bands. Journal of Experimental Psychology: Human Perception \& Performance, 17, 986-996.

Miskiewicz, A., BuUs, S., \& Florentine, M. (1994). Auditory facilitation: Procedural or sensory effect? Journal of the Acoustical Society of America, 96, 1429-1434.

Moore, B. C. J., \& Glasberg, B. R. (1987). Formulae describing frequency selectivity as a function of frequency and level and their use in calculating excitation patterns. Hearing Research, 28, 209-225.

Nieder, B., Buus, S., Cazals, Y., \& Scharf, B. (2007). Loudness reduction induced by a contralateral tone. Journal of the Acoustical Society of America, 122, 35-37.

NiEder, B., BuUs, S., Florentine, M., \& Scharf, B. (2003). Interactions between test- and inducer-tone durations in induced loudness reduction. Journal of the Acoustical Society of America, 114, 2846-2855.
Parker, S., \& Schneider, B. (1994). The stimulus range effect: Evidence for top-down control of sensory intensity in audition. Perception \& Psychophysics, 56, 1-11.

Poulton, E. C. (1989). Bias in quantifying judgments. Hillsdale, NJ: Erlbaum.

Scharf, B., BuUs, S., \& Nieder, B. (2002). Loudness enhancement: Induced loudness reduction in disguise? [Letter]. Journal of the Acoustical Society of America, 112, 807-810.

SCHNEIDER, B., \& PARKER, S. (1990). Does stimulus context affect loudness or only loudness judgments? Perception \& Psychophysics, 48, 409-418.

SuZUKI, Y., \& TAKeshima, H. (2004). Equal-loudness-level contours for pure tones. Journal of the Acoustical Society of America, 116, 918-933.

WAGNER, E., \& SCHARF, B. (2006). Induced loudness reduction as a function of exposure time and signal frequency. Journal of the Acoustical Society of America, 119, 1012-1020.

WARD, L. M., ARMSTrong, J., \& GolestANi, N. (1996). Intensity resolution and subjective magnitude in psychophysical scaling. Perception \& Psychophysics, $\mathbf{5 8}, 793-801$.

Zwicker, E., \& FASTL, H. (1990). Psychoacoustics: Facts and models. Berlin: Springer.

(Manuscript received March 12, 2007; revision accepted for publication December 18, 2007.) 